\title{
Valeurs exceptionnelles de fonctions transcendantes
}

\author{
P.A. DESROUSSEAUX, CNRS - UMR 8524, Mathématiques, bâtiment M2, \\ Université des Sciences et Technologies de Lille, 59655 Villeneuve d'Ascq Cedex, France \\ Courriel : pierre-antoine.desrousseaux@agat.univ-lille1.fr
}

Résumé - Dans le cas des fonctions hypergéométriques d'Appell -qui généralisent celles de Gauss- on construit un ensemble $\mathcal{E}_{1}$ permettant de faire le lien entre des valeurs algébriques de cette fonction en des points algébriques et des variétés abéliennes de la même classe d'isogénie -même si cet aspect géométrique n'apparait pas dans l'énoncé de ces résultats, c'est un outil essentiel pour ce travail-. Les propriétés liées à cet ensemble et le théorème 2 permettent d'extraire un contre-exemple à une conjecture de Coleman qui affirme qu'il n'existe qu'un nombre fini de classes d'isomorphisme de courbes algébriques de genre supérieur à quatre dont la jacobienne est à mulitplication complexe. On donnera également des conditions pour obtenir des valeurs transcendantes de ces fonctions hypergéométriques en des points algébriques.

\section{Exceptional values of transcendental functions}

Abstract - In the case of Appell hypergeometric functions -which generalize the Gauss hypergeometric function- one construct a set $\mathcal{E}_{1}$ which makes the link between algebraic values of Appell's functions at algebaric points and abelian varieties of the same isogeny class -even if the geometric aspect doesn't appear in these results, this is the main tool of this work-. Using properties of this set, one find as a consequence of the theorem 2, a counter-example of a conjecture of Coleman about finitness of the number of isomorphic class of algebraic curves with genus greater than four, for which jacobian as complex multiplication. One also give conditions to obtain transcendental values of these functions at algebraic points.

\section{Introduction.}

Lorsqu'on dispose d'une fonction transcendante (convenablement normalisée), on s'attend à ce qu'elle prenne très souvent des valeurs transcendantes en des points algébriques. On nomme donc valeur exceptionnelle ou point exceptionnel, un point alg'ebrique en lequel une telle fonction prend une valeur algébrique. L'ensemble exceptionnel est un ensemble de points exceptionnels, mais outre cette description arithmétique -à l'aide de la fonction trancendante considérée-, il doit posséder également une description géométrique -en terme de variétés abéliennes de la même classe d'isogénie-.

Un tel ensemble a été étudié par P. Cohen, J. Wolfart et G. Wüstholz, dans le cas de la fonction hypergéométrique de Gauss. Dans le premier paragraphe, on rappelle quelques propriétés relatives à cette fonction et notamment le "théorème de l'ensemble exceptionnel" obtenu dans [CoWü] traitant de la finitude de l'ensemble exceptionnel considéré. On terminera ce paragraphe en donnant un résultat sur la transcendance de valeurs de fonctions hypergéométriques de Gauss en des points algébriques (théorème 1).

L'objet principal est d'obtenir des résultats analogues dans le cas des fonctions hypergéométriques d'Appell qui généralisent -en deux variables- celles de Gauss, c'est l'objet des théorèmes 2 et 3. On en déduira ensuite quelques propriétés.

Ces travaux sont soumis à publication, pour une démonstration des résultats, on peut se référer à $[$ Des]. 


\section{La fonction hypergéométrique de Gauss}

Dans leurs travaux, J. Wolfart (1988), P. Cohen-J. Wolfart (1990) et P. Cohen-G. Wüstholz (1999) s'intéressaient à la finitude de l'ensemble

$$
\mathcal{E}:=\{x \in \overline{\mathbb{Q}}: F(a ; b ; c ; x) \in \overline{\mathbb{Q}}\}
$$

où $F$ est la fonction hypergéométrique de Gauss, c'est à dire pour :

$$
c \in \mathbb{Q} \cap] 0 ; 1[, \quad a ; b \in \mathbb{Q} \cap] 0 ; c[,
$$

et pour tout $x \in \mathbb{P}_{1}(\mathbb{C}) \backslash\{0 ; 1 ; \infty\}$,

$$
F(a ; b ; c ; x)=B(a ; c-a)^{-1} \cdot \int_{1}^{\infty} u^{b-c} \cdot(u-1)^{c-a-1} \cdot(u-x)^{-b} \cdot d u
$$

La fonction hypergéométrique de Gauss (tout comme la fonction hypergéométrique d'Appell que l'on étudiera par la suite) est une fonction transcendante (sauf pour certains cas particuliers de coefficients $a, b$ et $c$ ).

Il est naturel de se poser la question de la finitude de cet ensemble $\mathcal{E}$. La détermination de la finitude de cet ensemble, qui parachève les travaux de J. Wolfart, est dûe à P. Cohen et G. Wüstholz [CoWü] de 1999, qui s'appuie sur la version faible d'une conjecture d'André-Oort démontrée par B. Edixhoven et A. Yafaev [EdYa] ou [Ya]. Le résultat est le suivant :

THÉORÈME DE L'ENSEMBLE EXCEPTIONNEL :

(P. Cohen - J. Wolfart - G. Wüstholz - B. Edixhoven - A. Yafaev)

Pour $1-c>0$, Card $\mathcal{E}=\infty$ si et seulement si $\Delta(a ; b ; c)$ est arithmétique.

où $\Delta(a ; b ; c)$ est le groupe de monodromie lié à la fonction hypergéométrique de Gauss. A l'aide des outils utilisés et des travaux de H. Shiga et J. Wolfart, voir [ShWo], on peut démontrer :

THÉORÈME $1:$ Pour tout $x \in \overline{\mathbb{Q}} \backslash\{0 ; 1\}$,

si $F(a ; b ; c ; x)$ algébrique alors $F(b+1-c ; a+1-c ; 2-c ; x)$ est transcendant.

Remarque : grâce aux travaux de F. Beukers et J. Wolfart [BeWo], ou encore de N. Archinard [Ar], on connait des valeurs algébriques de fonctions hypergéométriques de Gauss (pas uniquement pour le groupe modulaire $S L_{2}(\mathbb{Z})$ ). Ce résultat donne donc des valeurs transcendantes explicites.

\section{La fonction hypergéométrique d'Appell}

On cherche à trouver un analogue de ces résultats dans le cas des fonctions hypergéométriques d'Appell. Les fonctions hypergéométriques d'Appell sont une généralisation au cas de deux variables des fonctions hypergéométriques de Gauss. Là encore, sauf certaines conditions particulières sur les coefficients $a, b, b^{\prime}$ et $c$, c'est une fonction transcendante, que l'on peut écrire sous forme intégrale de la façon suivante, pour des paramètres $a, b, b^{\prime}, c$ tels que :

$$
c \in \mathbb{Q} \cap] 0 ; 1\left[, \quad a ; b ; b^{\prime} \in \mathbb{Q} \cap\right] 0 ; c[,
$$


et pour tout $(x ; y) \in \mathcal{Q}:=\mathbb{P}_{1}(\mathbb{C})^{2} \backslash\{x ; y=0 ; 1 ; \infty ; x=y\}$,

$$
F_{1}\left(a ; b ; b^{\prime} ; c ; x ; y\right)=B(a ; c-a)^{-1} \cdot \int_{1}^{\infty} u^{b+b^{\prime}-c} \cdot(u-1)^{c-a-1} \cdot(u-x)^{-b} \cdot(u-y)^{-b^{\prime}} \cdot d u
$$

d'après [WoWü] et [CoWo], on peut considérer la fonction hypergéométrique d'Appell comme quotient de périodes non nulles de première espèce de variétés abéliennes. C'est cette description qui va permettre de faire le lien entre l'aspect arithmétique et l'aspect géométrique du problème.

La première difficulté consiste à construire l'ensemble exceptionnel relatif aux fonctions hypergéométriques d'Appell, on doit faire apparaitre une deuxième condition, non triviale, car contrairement au cas d'une variable, la seule condition d'algébricité portant sur la fonction étudiée ne suffit pas à donner une description géométrique à l'ensemble cherché. On obtient l'ENSEMBLE EXCEPTIONNEL suivant :

$$
\mathcal{E}:=\left\{(x ; y) \in \overline{\mathbb{Q}}^{2} \cap \mathcal{Q}:\left\{\begin{array}{l}
F_{1}\left(a ; b ; b^{\prime} ; c ; x ; y\right) \in \overline{\mathbb{Q}} \\
\left.F_{1}\left(b ; c-a ; 1-b^{\prime} ; c-b^{\prime} ; \frac{x}{x-1} ; \frac{x}{x-y}\right) \in \overline{\mathbb{Q}}\right\} .
\end{array}\right.\right.
$$

Pour l'analogue du théorème de l'ensemble exceptionnel cité précédemment, on s'intéresse à la distribution des variétés abéliennes à multiplication complexe, isogènes à une variété abélienne fixée. On obtient le résultat suivant, qui utilise la version faible d'une conjecture d'André-Oort, encore non-démontrée dans le cas des surfaces :

THÉORÈme 2: On suppose que la conjecture faible d'André-Oort dans le cas d'une surface est vraie. Soit $\Delta\left(\mu_{i}\right)$ d'action discontinue sur $B_{2}$. L'image de $\mathcal{E}$ est Zariski dense dans $B_{2} / \Delta\left(a ; b ; b^{\prime} ; c\right)$ si et seulement si le groupe $\Delta\left(a ; b ; b^{\prime} ; c\right)$ est arithmétique.

où $\Delta\left(a ; b ; b^{\prime} ; c\right)$ est le groupe de monodromie du système d'équations différentielles lié à la fonction $F_{1}$.

En illustration de ce résultat, du sens connu de la conjecture d'André-Oort et en utilisant le théorème 2 , on extrait dans la proposition suivante, un contre-exemple à une conjecture de Coleman qui affirmait qu'il n'existe qu'un nombre fini de classes d'isomorphisme de courbes algébriques de genre $g \geq 4$ dont la jacobienne est à multiplication complexe.

Proposition : Il existe une infinité de couples $(x ; y)$ de $\overline{\mathbb{Q}}^{2}$ pour lesquels la jacobienne de la courbe algébrique : $\mathcal{Y}(x ; y): v^{5}=u(u-1)(u-x)(u-y)$ de genre $g=6$, est à multiplication complexe.

Remarque : c'est le premier contre-exemple en dimension 2. Dans le cas de la dimension 1, deux autres contre-exemples ont été trouvés par J. de Jong et R. Noot, voir [dJN].

Enfin, le dernier théorème indique sous quelles conditions des valeurs algébriques de fonctions hypergéométriques d'Appell, en des points algébriques, conduisent à la transcendance d'autres valeurs de fonctions d'Appell. 
ThÉorème $3:$ Pour tout $(x ; y) \in \overline{\mathbb{Q}}^{2} \cap \mathcal{Q}$,

$$
\begin{aligned}
& \left\{\begin{array}{l}
F_{1}\left(a ; b ; b^{\prime} ; c ; x ; y\right) \in \overline{\mathbb{Q}} \\
F_{1}\left(b ; c-a ; 1-b^{\prime} ; c-b^{\prime} ; \frac{x}{x-1} ; \frac{x}{x-y}\right) \in \overline{\mathbb{Q}}
\end{array}\right. \\
& \text { entraîne }\left\{\begin{array}{l}
F_{1}\left(1-b ; a+1-c ; b^{\prime} ; 2+b^{\prime}-c ; \frac{x}{x-1} ; \frac{x}{x-y}\right) \notin \overline{\mathbb{Q}} \\
F_{1}\left(1-b^{\prime} ; b ; a+1-c ; 2+b-c ; \frac{y}{y-x} ; \frac{y}{y-1}\right) \notin \overline{\mathbb{Q}} \\
F_{1}\left(2-a ; 1-b ; 1-b^{\prime} ; 3-c ; x ; y\right) \notin \overline{\mathbb{Q}} \\
F_{1}\left(1-a ; 1-b ; 1-b^{\prime} ; 2-c ; x ; y\right) \notin \overline{\mathbb{Q}}
\end{array}\right.
\end{aligned}
$$

\section{Bibliographie :}

[AK] APPELL-KAmpé DE FÉRIET, Fonctions hypergéométriques et hypersphériques. Polynômes d'Hermite, L.M. (1926).

[Ar] N. ArChinaRD, Hypergeometric abelian varieties and identities, Thèse de Doctorat, Juin 2000.

[BeWo] F. Beukers-J. Wolfart, Algebraic values of hypergeometric functions, New Advances in Transcendence Number Theory, C.U.P. (1988), Proceedings of the 1986 Durham Symposium in Transcendental Number Theory, ed. A. Baker.

[CoWo] P. Cohen-J. Wolfart, Fonctions hypergéométriques en plusieurs variables et espaces de modules de variétés abéliennes, Ann. Scient. E.N.S (1993) pp665-690.

[CoWü] P. Cohen-G. Wüstholz, Application of the André-Oort conjecture to some questions in transcendance, Panorama of Number Theory (1999), ed. G. Wüstholz, C.U.P.

[Des] P.A. Desrousseaux, Valeurs exceptionnelles de fonctions transcendantes, Thèse doctorale. [dJN] J.DE JonG-R.Noot, Jacobians with complex multiplication, Arithmetic algebraic geometry, G. van der Geer-F. Oort-J. Steendbrink, eds, PM89, Birkhaüser (1991) pp177-192.

[EdYa] B. Edixhoven-A. Yafaev, Subvarieties of Shimura varieties,

Prépublication de l'I.R.M. de Rennes, Mai 2001.

[ShWo] H. Shiga-J. Wolfart, Criteria for complex multiplication and transcendance properties of automorphic functions, J. Reine Angew. Math. (1995) Vol.463 pp1-25.

[Wo] J. Wolfart, Werte hypergeometrischer Funktionen, Invent. Math. (1988) Vol.92 pp187-216.

[WoWü] J. Wolfart-G. WÜstholz, Der Überlagerungsradius gewisser algebraischer Kurven und die Werte der Betafuktion an rationalen Stellen, Math. Ann. (1985) Vol.273 pp1-15.

[Ya] A. YAfaev, Sous-variétés des variétés de Shimura, Thèse de Doctorat, Université de Rennes 1. 